\title{
STOMATOLOGY
}

UDC 616.37-002:577.125

\section{Comparative assessment of laser therapy efficiency of chronic generalized periodontitis treatment}

\author{
V. V.Akimov', A. S. Fedoskina ${ }^{2}$, D. A. Kuzmina 1 , T.I. Vlasova ${ }^{2}$ \\ ${ }^{1}$ St. Petersburg State University, \\ 7-9, Universitetskaya nab., St. Petersburg, 199034, Russian Federation \\ ${ }^{2}$ National Research Mordovia State University, \\ 68, Bolshevistskaya ul., Saransk, 430005, Republic of Mordovia, Russian Federation
}

For citation: Akimov V.V., Fedoskina A.S., Kuzmina D. A., Vlasova T. I. Comparative assessment of laser therapy efficiency of chronic generalized periodontitis treatment. Vestnik of Saint Petersburg University. Medicine, 2020, vol. 15, issue 3, pp. 198-202. https://doi.org/10.21638/spbu11.2020.304

This paper is a result of collaboration between personnel of Saint-Petersburg and Saransk medical universities. The purpose of the investigation was to improve treatment and secondary prevention of chronic generelized periodontitis on the base of pathogenetically substantiated scheme of laser therapy. Cohort prospective study included 98 patients $(31$ men and 67 women) suffered from chronic generelized periodontitis at the age from 30 to 50 years with disease prescription from 3 to 10 years. All the patients were divided into three groups: standard anti-inflammatory therapy, laser therapy, antioxidant therapy. Efficiency of periodontitis treatment in the groups was estimated by clinical laboratory data, stomatological indices (PMA, SBI, API, ) resistance of gingival capillaries; degree of osseous resorption. State of lipid peroxidation (LP) was estimated by the level of malondialdehyde (MDA, $\mathrm{Fe}^{2+}$-MDA) and phospholipase $\mathrm{A}_{2}$. It was found that application of laser and metabolic therapy considerably increase efficiency of standard treatment regimen and secondary prevention of chronic periodontitis, that is manifested in significant decrease structural-functional changes and in restoration of paradontium tissues microcirculation.

Keywords: chronic generelized periodontitis, laser therapy.

Actuality. Frequency of pathological paradontium tissues changes dramatically increased together with civilization development, and in Russia it reached 95-100\% [1-3]. Such a comprehensive prevalence and treatment complexity stipulates importance of knowledge of this pathology in routine work not only of parodontist, but of general practise stomatologist as well. The most hard and the most prevalence paradontium pathol-

(C) St. Petersburg State University, 2020 
ogy is chronic generalized periodontitis. Availability of tremendous amount of schemes and methods of combined treatment does not remove actuality of this problem in recent stomatology [4-9]. Stated above reasons determines prevention and treatment of chronic generalized periodontitis improvement $[10 ; 11]$.

Purpose of the work. The purpose of the study was to improve treatment and secondary prevention of chronic generelized periodontitis on the base of pathogenetically substantiated scheme of laser therapy.

\section{Materials and methods of investigation}

Cohort prospective study included 98 patients ( 31 men and 67 women) suffered from chronic generelized periodontitis of medium severity at the age from 30 to 50 years with disease prescription from 3 to 10 years. Patients were divided into three groups comparable by the age, gender and disease severity. All the patients underwent complete physical examination at admission, at sixth day of treatment, twelfth day on therapy completion, after 6 months after course of therapy.

In the first control goup $(n=33)$ patients receive standard anti-inflammatory therapy: after performing professional hygiene of oral cavity, suspension of chlorohexidine with metrogyl was loaded into deep periodontal pockets, oral bath with dioksidin or dimexidum were carried out, salve dressing with anti-inflammatory ointment (Lingesin, Metrogyl denta, Butadion, Cholisal) were applied, anti-inflammatory therapy in the form of antimicrobial agents (flagyl, Kliostom, Metrogyl) was carried out, desensitizing preparations (diazolin), vitamins $\mathrm{A}, \mathrm{C}, \mathrm{P}$ were assigned and nonsteroidal anti-inflammatory preparations, usually indometacin.

Patients in the second group $(\mathrm{n}=32)$ additionally to basic therapy received laser therapy. With this aim appliance "Matrix" was used. Irradiating was performed using KLO3 radiating head (wavelength $635 \mathrm{~nm}$ ) with radiation power $10 \mathrm{~mW}$. Alveolaris processus was irradiated during 2-5 minutes (impact time increases by increment 30 seconds per day, maximal exposure time constituted 5 minutes). Laser therapy sessions were carried out daily in course of 12 days.

Patients in the third group ( $\mathrm{n}=33$ ), along with traditional therapy and sessions of laser therapy using indicated above scheme, during 12 days received daily additional antioxidative preparation Mexicor.

Efficiency of periodontitis therapy in the groups was estimated by clinical-laboratory data before and after treatment, as well as after each of 6 after therapy course. All the patients underwent complete physical examination: stomatological, clinical-laboratory, roentgenological, biochemical and functional examination before and after the treatment.

For objective estimation of periodontal tissues condition the following clinical-functional dental indices were used: PMA — papillary marginal alveolar index; SBI — sulcus bleeding index; API (Approximal plaque index) - index of approximal tooth surfaces hygiene; by dosed vacuum method (by V. I. Kulazhenko) resistance of gum capillaries was studied; by condition of partitions of bone separating the alveoli of different teeth the degree of osseous resorption was determined. Radiological diagnostic method was used for obtaining orthopantomogram and intra-oral X-ray pattern of teeth and jaws in occlusion. Rate of bone tissue resorption was determined by of partitions of bone separating the alveoli of different teeth. 
State of lipid peroxidation (LP) was estimated by the level of secondary products malondialdehyde (MDA, $\mathrm{Fe}^{2+}$-MDA) in blood plasma of patients. Registration of catalytic phospholipase $\mathrm{A}_{2}$ activity was conducted depending on free fatty acids formation, detected by potentiometric method in the medium containing 10 millimole of tris-acetate buffer ( $\mathrm{pH}$ 8.0), 150 millimole of triton $\mathrm{X}-100,10$ millimole of $\mathrm{CaCl}_{2}$ and substrate (1.2 millimole of egg yolk phosphatidylcholine). Catalase activity was studied using spectrophotometric method, which is based on peroxides ability to form with ammonium molybdate stable colored complex. Obtained digital data were processed using method of variation statistics with Student's test application.

\section{Results of investigations and their discussion}

In course of investigation it was revealed that at chronic generalized periodontitis of medium severity oral health status in patients before therapy was estimated by API index as unsatisfactory. Dynamics of papillary marginal alveolary index (PMA index) evidenced about inflammation of gingival membrane. After performing complete therapy PMA index decreased by $22.74-45.02 \%(\mathrm{p}<0.05)$. By the end of therapy decreasing of hygiene index (API) bu 33.72\% ( $\mathrm{p}<0.05)$ was fixed. Degree of gums bleeding (SBI index) against the background of conventional therapy were also decreased only by the end of therapy by $57.11 \%(\mathrm{p}<0.05)$. In course of determining functional status of parodont vascular it was found that the time of haematoma formation in gum was elongated by $55.81-107.70 \%$ $(\mathrm{p}<0.05)$.

The studies demonstrated that at chronic generalized periodontitis in $75 \%$ of patients intensification of free-radicals processes and activation of phospholipase A2 in erythrocytes was noticed, that witnesses significance of peroxidation processes in disease process. It was fixed a considerable increase in malonyldialdehyde content (MDA) and induced MDA by 86.02 and $27.19 \%(\mathrm{p}<0.05)$ respectively. Before treatment catalase activity indicator exceeded the norm by $14.29 \%(\mathrm{p}<0.05)$. Index of erythrocytic phospholipase A2 activity exceed the norm by $55.61-74.70 \%(\mathrm{p}<0.05)$ at all the stages of dynamic observation. Intensity of lipid peroxidation and fermentative activity in erythrocytes against the background of conventional therapy decreases, but did not reach original level (figure).

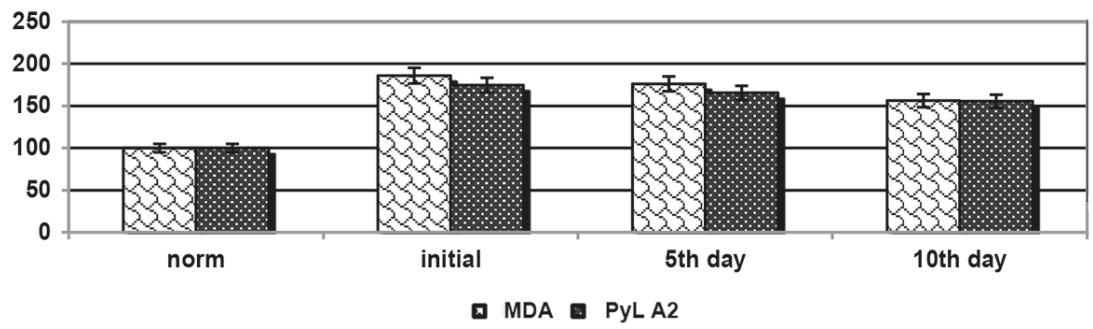

Fig. Dynamics of MDA level and phospholipase A2 activity

Note: All the indicator changes are definit in respect of norm.

At chronic periodontitis meta-stabilizing phenomena occurred in $71.77 \%$ of patients. So, before performing therapy content of total phosphotides was lewer than norm by $9.23 \%(\mathrm{p}<0.05)$, at that in its spectrum considerable increase of lysoform fractions and phosphatidyl serine was noted, which exceeded the norm by 74.41 and $32.36 \%(\mathrm{p}<0.05)$ 
respectively. Indicators of cholesterol esters free fatty acids also exceeded the norm. Against background of conventional therapy it is necessary to note favorable dynamics of studied indicators, nevertheless even at the tenth day of therapy most of them did not reach normal level.

According to the stated above data it become obvious, that conventional therapy must be replenished by pharmacological preparations, possessing ability to correct noted pathological changes in homeostasis system.

In the second and in the third groups of clinical studies estimation of additional application of quantum therapy and complete therapy (laser therapy + Mexicor) in treatment and prevention of chronic periodontitis of medium severity recurrence.

It was found out that application of complete therapy with inclusion antioxidant Mexicor and quantum irradiation promoted decrease of oxidative processes evidence on a local and system levels as well, that was demonstrated by decrease of lipid peroxidation products and recovery of antioxidant ferments activity in saliva and in blood plasma as well. So, by the twelfth day of dynamic observation, the level of malonyldialdehyde in blood plasma of patiets with chronic periodontitis by the end of therapy was lower than in control by $15.83 \%(\mathrm{p}<0.05)$, did not significantly differs from the norm. Superoxide anion scavenger activity а в within the normal values and was above the cjntrol value by $22.75 \%$ $(\mathrm{p}<0.05)$. Phospholipase $\mathrm{A}_{2}$ activity by the end of treatment was lower than control data by $13.40 \%(\mathrm{p}<0.05)$, decreasing to norm. Similar positive changes were registered and at estimation of oxidative phenomena in saliva by the end of remedial measures decreasing of TBA-reactive substances was registered by $28.57 \%(\mathrm{p}<0.05)$ as compared with control group. Superoxide anion scavenger activity at second stage of observation was lower than control data by $17.01 \%(\mathrm{p}<0.05)$ and reached reference values.

Application of only photon therapy in treating chronic moderate severity periodontitis also demonstrated improvement of oxidative stress markers. Decrease of free radical reactions activity in organism was revealed along with recovery of reserve enzymatic antioxidant, particularly SOD. It should be noted that effect of laser therapy was less expressed in correction of oxidative stress markers, than in case of complete therapy application with Mexicor inclussion.

In conclusion it is necessary to note that obtained results proves the efficiency of laser and metabolic therapy not only in treating, but also in prevention of chronic periodontitis recurrence.

\section{Conclusions}

1. Efficiency of standard anti-inflammatory therapy of chronic genereluzed periodontitis is insufficient because it often lead to recurrences of the disease.

2. Application of laser therapy increases efficiency of standard scheme of treatment and secondary prention of chronic genereluzed periodontitis.

\section{References}

1. Lukinykh L. M. Diseases of the oral cavity. Nizhnii Novgorod, NGMA Publ., 2004. 508 p. (In Russian)

2. Chunikhin A. A., Bazikyan E. A., Zayratyants O.V. Estimation of the effectiveness nanosecond laser therapy of periodontal diseases in the experiment. Rossiiskaia stomatologiia, 2017, vol. 10, no. 4, pp. 3-7. (In Russian) 
3. Cobb C.M. Lasers and the treatment of periodontitis: the essence and the noise. Periodontol. 2000, 2017, vol. 75, no. 1, pp. 205-295.

4. Gazhva S. I., Shkarednaia O. V., Piatova E.D. Integrated approach to the treatment of oral mucosa diseases in patients with chronic gastritis. Stomatology, 2013, vol. 65, pp. 16-19. (In Russian)

5. Navruzova U. O. Features of the periodontal disease at the metabolic disorder. Biology and integrative medicine, 2019, vol. 2, pp. 28-42. (In Russian)

6. Orekhova L. Yu., Loboda E. S., Oboeva M. L. Photodynamic therapy in complex treatment of inflammatory periodontal diseases. Actual problems of laser medicine, 2016, pp. 171-181. (In Russian)

7. Bartold P.M. Lifestyle and periodontitis: The emergence of personalized periodontics. Periodontol. 2000, 2018, vol. 78, no. 1, pp. 7-11.

8. Gulati N. N., Masamatti S. S., Chopra P. Association between obesity and its determinants with chronic periodontitis: A crosssectional study. J. Indian Soc. Periodontol., 2020, vol. 24, no. 2, pp. 167-172.

9. Fernandes F. L. A., Clin J. Treatment of experimental periodontal disease by photodynamic therapy in immunosuppressed rats. Periodontol., 2009, vol. 36, no. 3, pp. 219-228.

10. Amkhadova M.A., Kopetskiy I.S., Prokopev V.V. The effectiveness ofphotodynamic therapy in the treatment of chronic generalized periodontitis. Rossiyskii stomatologicheskii zhurnal, 2016, vol. 20, no. 1, pp. 12-15. (In Russian)

11. Bazikjan E. A., Syrnikova N. V., Chunihin A.A. Perspective laser technologies in the therapy of periodontal diseases. Parodontologiia, 2017, vol. 22, no. 3, pp. 55-59. (In Russian)

Received: November 9, 2020

Accepted: December 15, 2020

Authors' information:

Vladimir V.Akimov — Postgraduate Student; akimov.spbmapo@mail.ru

Anna S. Fedoskina - Postgraduate Student; anfedoskina@yandex.ru

Diana A.Kuzmina - Dr. Sci. (Medicine), Associate Professor; dianaspb@rambler.ru

Tatiana I. Vlasova - Dr. Sci. (Medicine), Professor; vap.61@yandex.ru 